\title{
Fehler-Planungen
}

\section{Zur Geschichte und Theorie der industriellen Obsoleszenz}

\author{
VON MARKus KRAJEWSKI
}

"We could make lamps so that they would last one thousand years,
but nobody would want to use them."
(Interne Phoebus-Kommunikation)

\section{Überblick}

Der Artikel untersucht den Zusammenhang von industrieller Produktion und künstlicher Begrenzung der Lebensdauer von Produkten, besser bekannt unter dem Begriff der geplanten Obsoleszenz. Am Beispiel der Glühlampe geht es in dieser diskursanalytischen Untersuchung erstens darum, die Schwierigkeiten, das Ableben einer Technik gezielt in der Produktion festzulegen, mit den ökonomischen Kontexten und Vorstellungen der Zeit in Verbindung zu bringen, in denen ein neues Produktions- und Verwertungsparadigma formuliert wird: Dieses setzt ab dem frühen 20. Jahrhundert darauf, koordinierte Forschungsanstrengungen nicht dafür einzusetzen, um das technisch-materiell mögliche Optimum an Haltbarkeit und Nutzungsdauer zu erreichen, sondern es setzt an dessen Stelle ein genau kalkuliertes ökonomisches Optimum, das einer systematischen Verkürzung von Lebenszeiten gleichkommt. Im Fall der Glühlampe wurde sogar für eine verlässliche Dysfunktionalität der Technik nach 1.000 Stunden Brenndauer gesorgt. Zweitens wird erörtert, was dieser Fall für gängige Annahmen und Theorien zur Fertigung und Erkenntnisproduktion im (Industrie-)Labor sowie zum industriellen Fehler bedeutet.

\begin{abstract}
This article explores the relationship between industrial production and artifical limitations within the life cycle of products, which is also known under the term ,planned obsolescence'. Taking the light bulb as an example, this discourse analysis focuses on the difficulties attending the implantation of technical failure as they relate to the economical contexts and new paradigms in mass production in the early twentieth century. Coordinated research efforts tend to reach an agreed economical optimum rather than the feasible technical optimum based on sustainability aims. In the case of the light bulb, this economical optimum prescribed a product lifetime of precisely 1,000 hours which the electrical companies had to meet to avoid fixed fines controlled by the Phoebus cartel. Furthermore, this article discusses how these prescriptions
\end{abstract}


require a reverse research strategy that aims to de-optimize an efficient product, and how this strategy affects the notion of technical errors.

Worin die Gemeinsamkeiten einer handelsüblichen Schmerztablette, einer Tafel Schokolade und einer Getränkedose liegen, wird spätestens auf den zweiten Blick klar. Es liegt direkt vor Augen: Sowohl die Tablette, eine Schokoladentafel und die Öffnung einer Getränkedose zeichnen sich jenseits ihrer Wirk- und Inhaltsstoffe durch einen gut sichtbaren Mechanismus aus, den man gemeinhin als eine Sollbruchstelle bezeichnet. Was diese drei Alltagsgegenstände also eint, ist eine vorgefertigte Schwäche im Material, eine Verdünnung der Stärke an zuvor definierter Stelle, die bei Belastung zu einer kontrollierten Bruchkante führt und den Gegenstand in zwei Teile zerlegt. Wird das Objekt insgesamt höher belastet, als die schwächste Stelle verträgt, versagt genau diese vorgefertigte Zone ihren Zusammenhalt. Das Wirkungsprinzip folgt dem Zweck, infolge der Lädierung einen Bruch herbeizuführen, der verglichen zu möglichen Folgen für das Gesamtsystem den Schaden möglichst gering und kontrollierbar hält.

Industriell gefertigte Gegenstände leben kaum ewig. Ihre Lebensdauern sind begrenzt, sei es durch Entropie, durch Verbrauch oder durch allmählichen Verschleiß. Manche, wie die Schokoladentafel, vergehen durch Konsumtion, andere werden durch eine allmähliche Abnutzung mit der Zeit unbrauchbar. Doch diese Zeitspanne kann je nach Gegenstand lange dauern, länger jedenfalls, als es den Produzenten des Gegenstands behagen mag, weil ein Produkt beim Verbraucher jene Lücke wirksam ausfüllt, die vom Produkt des Herstellers im Lagerregal erneut besetzt werden könnte. Was liegt also näher, als auch diese nur langsam alternden Gegenstände mit einer Sollbruchstelle auszustatten, obwohl sie aus Sicht des Verbrauchers gar nicht brechen sollten.

So wie bereits in der Natur mit ihrem langen evolutiven Entwicklungsgang Sollbruchstellen auftauchen, etwa bei Bruch-Weiden in Flussauen, findet sich dieses Prinzip ebenso bei komplexeren technischen Systemen, sei es bei Schmelzsicherungen in elektrischen Stromkreisen, sei es als mechanische Elemente, etwa bei einem Seil zwischen Motor- und Segelflugzeug. In all diesen Fällen ist eine eigens vorgesehene oder gefertigte Schwachstelle so ausgelegt, dass sie ab einer bestimmten Beanspruchung ihren Zusammenhalt aufgibt und an der beabsichtigten Stelle bricht. Mit diesem kontrollierten Aussetzen des Betriebs soll verhindert werden, dass das System an anderer, schwierig oder nicht kontrollierbarer Stelle überbeansprucht wird und infolgedessen mehr als nur die kleine vorgefertigte Bruchstelle in Gefahr gerät, irreparable Schäden oder gar die Auflösung des Systems zur Folge zu haben.

Im Unterschied zu diesen mitunter essenziellen, weil unfallverhütenden Elementen technischer Systeme, aber auch im Unterschied zu Sollbruchstel- 
len bei Tabletten, Schokoladen und Verpackungen, die allesamt mit einer offenkundigen Vorrichtung dieser Art arbeiten, weil die Stellen als solche gut sichtbar sind, operiert eine andere Gruppe von Gütern: Darunter befinden sich ebenfalls technische Objekte, auch Alltagsgegenstände wie Spanplatten, Nylonstrümpfe, Zahnbürsten, Tintenstrahldrucker oder Unterhaltungselektronik, aus deren Bereich sich in letzter Zeit die Fallbeispiele mehren: So werden Kondensatoren an Stellen platziert, wo sie hohen Temperaturen ausgesetzt sind, welche ihre Lebensdauer verkürzen, oder digitale MP3-Player halten nur so lange wie das schwächste Glied, der nicht auswechselbare Akku. So kam es 2003 etwa zu einer Sammelklage mehrerer Nutzer des Apple-iPods gegen den Konzern, in dem dieser beschuldigt wurde, minderwertige Akkus zu verwenden, welche die Lebenszeit des Geräts deutlich senkten; Apple setzte daraufhin Kompensationen und auch einen Akku-Austauschdienst ein. ${ }^{1}$

In solchen Fällen sind Sollbruchstellen wirksam, die jedoch für den Konsumenten unwissentlich und unsichtbar eingearbeitet werden, um ihm tunlichst verborgen zu bleiben. Dies berührt bereits einen heiklen Punkt ihrer Untersuchung sowie ihrer Historisierung: Der Extremfall der geplanten Obsoleszenz, nämlich die versteckten Sollbruchstellen, die eine Lebensdauer des Produkts, vor allem aber seinen Tod bereits im Fabrikationsprozess mitzubestimmen versuchen, operiert im Opaken und ist nur in Ausnahmefällen offenzulegen, etwa wenn sie zum Gegenstand von Gerichtsverfahren wird. Kein Hersteller des 20. Jahrhunderts hat bisher freiwillig Details zur Auslegung von Lebensdauern offenbart, ganz im Gegensatz etwa zu anderen ökologischen Nutzungskriterien wie dem Energieverbrauch. Ohnehin fehlen technikhistorische Studien dazu, wie sich die Lebensdauern einzelner, so genannter "langlebiger“ Konsumgüter (,,consumer durables“) in mittelfristiger Perspektive geändert haben. Dabei tendiert bereits dieser Dachbegriff zum Oxymoron, denn solche „langlebigen“ Artefakte haben kurze Lebensdauern von ein bis sechs Jahren im Fall vieler Elektronikgeräte. ${ }^{2}$ Auch hat die Technikgeschichte bisher die Rolle von Lebensdauern in der Konstruktion noch nicht beleuchtet.

Die im Folgenden zu entwickelnde Historiografie des geplanten Verschleißes hat es daher nicht nur mit einer schwierigen Quellenlage zu tun. Die Opazität der Quellen wird von ihrer prinzipiellen Heterogenität sowie von einer nur mühsam zu überschauenden Sachlage weiter verkompliziert. Denn

1 Apple hatte in der Werbung versprochen, die Batterie halte so lange, wie der iPod lebe, was nicht zutraf. Vgl. Peter Cohen, Apple settles iPod battery class action suit, in: Macworld vom 2.6.2005, online unter: http://www.macworld.com/article/1045105/ipodsuit.html [Stand: 22.1.2014]. Inzwischen gibt es auch für das Problem der - weiterhin als an sich nicht auswechselbar designten - Akkus des iPods wie für viele andere Elektronikgeräte einen Online-Guide für das Do-it-Yourself-Basteln, um durch ein solches subversives Basteln die Nutzungsdauern zu verlängern, vgl. http://electronics.howstuffworks.com/how-to-tech/ how-to-replace-ipod-battery.htm [Stand 22.1.2014].

2 Zahlen nach: Timothy Cooper, Reflections on Product Life Spans and the "Throwaway Society", in: Journal of Industrial Ecology 9, 2005, S. 51-67. 
Obsoleszenz operiert am Schnittfeld von Technik und Wirtschaft; ökonomische Diskurse verschränken sich mit technischen und marketingstrategische mit konsumkritischen Debatten. Das Phänomen der geplanten Obsoleszenz ist unter leicht variierter Begrifflichkeit in vergleichsweise heterogener Ausprägung in verschiedenen wissenschaftlichen Diskursen, vor allem in der Ökonomie, der Jurisprudenz, in den Technik- und Ingenieurwissenschaften in der ersten Hälfte des 20. Jahrhunderts entwickelt worden. Ökonomische Lehrbücher listen, ausgehend von Gregory (1947), das Phänomen der geplanten Obsoleszenz als die künstliche Erzeugung eines Bedarfs, als die Schaffung eines Anreizes zum Neukauf, obwohl der Bedarf eigentlich gesättigt ist, weil das Gerät ja schließlich voll funktionstüchtig wirkt. Mit anderen Worten: In ökonomischen Abhandlungen erscheint Obsoleszenz als eine reine, wenngleich für den Verbraucher undurchsichtige Marketingstrategie, ohne darauf einzugehen, welcher fertigungstechnischer Anstrengungen das möglichst exakte Auslegen von Lebensdauern möglicherweise bedarf. Daneben wiederum existiert ein kultur- und konsumkritischer, populärer Diskurs zur geplanten Obsoleszenz, der die Herausbildung des westlichen Massenkonsums im 20. Jahrhundert in zentralen Etappen - seiner Herausbildung in der amerikanischen Zwischenkriegszeit, seiner Kritik in der Umweltbewegung der 1970er Jahre und der neuerlichen Diskussion um eine Postwachstumsgesellschaft - begleitet hat. Unternehmen selbst wiederum können, weil geplante Obsoleszenz am diffusen Schnittbereich von Technik, Ökonomie und Zeitgeist bzw. angenommenen Nutzer- und Modewünschen operiert, die Begrenzung von Lebensdauern über zahlreiche Facetten jenseits des Interesses nach einem schnelleren Absatz begründen, die wesentlich verbraucherfreundlicher klingen: So etwa mit dem Hinweis auf Kosteneffekte und jeweilige Kaufkraft der anvisierten Konsumentengruppe; auf konstruktionstechnische Erfordernisse oder neue Sicherheitsbestimmungen; im digitalen Zeitalter außerdem mit dem Hinweis auf die immer kürzer werdende Abfolge von Innovations- und Produktlebenszyklen - Produktgenerationen lösen sich hier teils in nur zwölf Monaten oder weniger $\mathrm{ab}$ - sowie dem Wunsch der Nutzer, Modelle zügig zu wechseln.

Das zentrale Beispiel für die folgende, methodisch der historischen Diskursanalyse verpflichteten Untersuchung liefert ein seinerseits schon fast historisch gewordener Gegenstand: die gute alte Glühlampe, mattiert oder nicht, mit mehr als 60 Watt oder dunkler, die aufgrund ihrer verwickelten Geschichte zum Pionier der industriellen Obsoleszenz, zum ersten Gebrauchsgegenstand mit technisch kontrollierbarem Verfallsdatum geworden ist. Mit ihr ist es gelungen, den Durchsatz an Leuchtmitteln des sogenannten Verbrauchers ${ }^{3}$ genauestens

3 Der, Verbraucher' umfasst eine vergleichsweise mächtige Semantik, die sich grundlegend in eine technische Ebene (Verbraucher im Stromkreis, also z.B. eine Glühlampe) und eine soziale Ebene differenzieren lässt, zu einer ersten soziologischen Einordnung vgl. Nepomuk Gasteiger, Der Konsument. Verbraucherbilder in Werbung, Konsumkritik und Verbraucherschutz 1945-1989, Frankfurt a.M. 2010. Bemerkenswert bleibt darüber hinaus, dass sich 
zu regeln. Die Glühlampe diente bereits in einigen marxistischen Kritiken der 1970er Jahre sowie in einem technikhistorischen Artikel von Günther Luxbacher zu Reparatur und Lebensdauer als Fallbeispiel der industriell induzierten Obsoleszenz; ${ }^{4}$ außerdem ist sie das Paradebeispiel populärer Abhandlungen, die im so genannten Glühlampen-Kartell eine Verschwörung zu entdecken glauben. Hier wie dort wurden die historischen Spuren zur Obsoleszenz allerdings nicht näher verfolgt: In der überlieferten Geschäftskorrespondenz des Glühlampenkartells lassen sich erstaunlich explizite Äußerungen zur Verkürzung der Lebensdauern dokumentieren. Diese werden im ersten Teil des Artikels als diskursive Ursprünge der geplanten Obsoleszenz vorgestellt, zu denen weitere Ideen eines absichtlichen Verschleißes in öffentlichen Debatten traten.

Was die bisherigen Diskussionen wie auch die historischen Arbeiten zur Strategie der geplanten Obsoleszenz allerdings kaum beleuchtet haben, ist der Fakt, dass es zusätzlicher Forschungsleistungen im Industrielabor bedarf, um sie verlässlich im Produkt umzusetzen. Das Produkt muss eine prognostizierbare Lebensdauer erhalten - oder umgekehrt formuliert: Sein Verfallsdatum muss zuverlässig ,mitkonstruiert“ werden. Dies setzt eine neuartige Industrieforschung voraus, wie sie überhaupt erst im Zuge der entstehenden Material- und Gebrauchswertprüfung sowie der statistischen Qualitätskontrolle seit Beginn des 20. Jahrhunderts möglich wurde. ${ }^{5}$ Die Labore der Glühlampenfabriken repräsentieren die ersten dafür eingerichteten Forschungsstätten der Elektroindustrie; einige technikhistorische Arbeiten haben die Leistungen von Industrieforschern und -mathematikern wie Pirani, Runge, Plaut oder Daeves herausgestellt, mit deren Hilfe etwa bei Osram eine fertigungsbegleitende statistische Qualitätskontrolle entwickelt wurde, um normierte Produkt-Qualitäten in der Massenproduktion um 1930 durchzusetzen. ${ }^{6}$ Dazu

das Verbrauchsgut oder Produkt überhaupt mit einem Versprechen von Langlebigkeit verbinden lässt, umfasst Verbrauch doch als Begriff allen voran eine aufzehrende, destruktive Komponente.

4 Vgl. Gerhard Bodenstein u. Hans Leuer (Hg.), Geplanter Verschleiß in der Marktwirtschaft, Frankfurt a.M. u. Zürich 1977; Günther Luxbacher, Die 1000-Stunden-Frage. Die andere Seite der Massenproduktion: Reparatur, Austauschbau, Lebensdauer und die Anfänge der Gebrauchswertforschung technischer Konsumgüter, in: Lars Bluma, Karl Pichol u. Wolfhard Weber (Hg.), Technikvermittlung und Technikpopularisierung. Historische und didaktische Perspektiven, Münster 2004, S.103-120.

5 Zur Gebrauchswertforschung in der Schuhproduktion vgl. Anne Sudrow, Der Schuh im Nationalsozialismus. Eine Produktgeschichte im deutsch-britisch-amerikanischen Vergleich, Göttingen 2010; für die Glühlampenindustrie vgl. Günther Luxbacher, Massenproduktion im globalen Kartell. Glühlampen, Radioröhren und die Rationalisierung der Elektroindustrie bis 1945, Berlin 2003.

6 Vgl. u.a. Luxbacher (wie Anm. 4); zu Runge außerdem: Renate Tobies, „Morgen möchte ich wieder 100 herrliche Sachen ausrechnen“: Iris Runge bei Osram und Telefunken, Stuttgart 2010. Pirani leitete zunächst das Forschungslabor im Berliner Glühlampenwerk von Siemens (1905-1919), später dann bei Osram die Wissenschaftlich-Technische Dienststelle für elektrische Beleuchtung (1919-1936), wo er ab 1921 auch als Direktor der Studiengesellschaft für elektrische Beleuchtung wirkte. Hubert Curt Plaut entwickelte während seiner Zeit bei 
gehörte auch die normierte Lebensdauer der Glühlampen. Es wurde aber noch nicht hinterfragt, inwiefern und wie die damaligen Industrielabore zugleich auch an der von der Industriespitze festgelegten Verkürzung der Brenndauern mitwirkten. Es wird Aufgabe einer technikhistorischen Untersuchung sein, die genauen Verflechtungen in den einzelnen Bereichen der Industrieforschung mit der geplanten Obsoleszenz sowie die Details der technischen Umsetzung der Sollbruchstelle in Labor und Fertigungsprozess nachzuverfolgen.

Die Stoßrichtung dieses Artikels zielt demgegenüber jedoch auf eine andere Perspektive, insofern sie eine wissenschaftstheoretische sowie kulturwissenschaftliche Reflexion unternimmt: Wenn Wissenschaft und Technik dafür eingesetzt werden, Lebensdauern nach einem ökonomischen Optimum - also nicht nach dem von Material, Technik und Fertigung her Möglichen - auszurichten, ohne dies transparent zu machen, so kommt dies einer fundamentalen Änderung im Paradigma des wissenschaftlich-technischen Schaffens oder zumindest seines Anspruches gleich. Geplante Obsoleszenz wird daher als eine zuverlässige Fehlerhaftigkeit gedeutet, die es industriell zu implantieren gilt, und es wird gezeigt, inwiefern sie gängige Annahmen nicht nur zum ,technischen Fortschritt“, sondern auch zum Erkenntnisprozess im (Industrie-)Labor sowie zum „Fehler“ in Frage stellt.

\section{Die Glühlampe, zum Beispiel}

Genf, 24. Dezember 1924. An einem dunklen Dezembertag, dem Vorabend zum Weihnachtsfest, versammeln sich am Ufer des Lac Léman einige verschwiegene Herren, um das ,Fest des Herrn' zu ihrem ,Fest des Lichts' zu machen. Eingefunden haben sich die führenden Vertreter von Osram (Deutschland), International (USA), Philips (Holland), Compagnie des Lampes (Frankreich), Tungsram (Ungarn) u.a., kurzum die Elite der glühlampenproduzierenden Konzerne $^{7}$ mit den Repräsentanten ihrer monopolisierten Nationalindustrien in abendmahlartiger Runde. Während anderenorts bereits Lichter liebevoll auf Zimmertannen gereiht werden, gründen die Herren Phoebus, indem sie an diesem Heiligen Abend den Entwicklungs- und Fortschrittsvertrag auf dem Gebiete der internationalen Glühlampenindustrie unterzeichnen mit dem Ziel, „,ie Zusammenarbeit aller Vertragsparteien sicherzustellen, zur Vorsorge

Osram (1920-1936) als Leiter der Abteilung Technische Statistik innovative Verfahren, die unter dem von Karl Daeves geprägten Titel ,Großzahlforschung s stochastische Methoden in die Industrieproduktion zur Analyse von Produktqualität und -kontrolle einführten.

7 Vgl. zur Geschichte der Vormachtstellung dieser Konzerne und zur Geschichte von Phoebus en detail Markus Krajewski, Vom Krieg des Lichtes zur Geschichte von Glühlampenkartellen, in: Peter Berz, Helmut Höge u. Markus Krajewski (Hg.), Das Glühbirnenbuch, Wien 2001, S.173-193. Im Anschluss daran auch Luxbacher (wie Anm. 5); ders. (wie Anm. 4). Bekannt geworden ist Phoebus vor allem durch Thomas Pynchons Roman Die Enden der Parabel von 1973, der sich wiederum auf die frühe Analyse von George W. Stocking u. Myron W. Watkins, Cartels in Action. Case Studies in International Business Diplomacy. The Twentieth Century Fund, New York 1947, stützte. 
für eine vorteilhaftere Ausnutzung ihrer Fabrikationsmöglichkeiten bei der Herstellung von Lampen, Sicherung und Aufrechterhaltung einer gleichmäBig hohen Qualität, Verbesserung der Wirtschaftlichkeit bei der Verteilung des Absatzes und Steigerung der Wirksamkeit elektrischer Beleuchtung und Erhöhung des Lichtverbrauches zum Vorteil des Verbrauchers" ${ }^{8}{ }^{8}$ Hinter diesen Sätzen verbirgt sich nicht nur das erste weltweit wirksame Kartell der Wirtschaftsgeschichte. ${ }^{9}$ Mehr noch, die Zwischenkriegszeit sollte zur Hochzeit internationaler Kartelle werden und Phoebus wirkte dabei als eines der bekanntesten und bestorganisierten Kartelle stilbildend. Und spätestens seit der auskunftsfreudigen Selbstbeschreibung ihres Initiators William Meinhardt (1932) kann keinesfalls behauptet werden, dass Phoebus im Geheimen gearbeitet habe. Vielmehr verbirgt sich dahinter vor allem eine strenge ökonomische Strategie, die viele bzw. vieles erreicht - nur sicherlich zum Vorteil des Verbrauchens und nicht eines menschlichen Verbrauchers, dem laut Zitat angeblich die Priorität eingeräumt wird.

Phoebus diente einerseits als Kontrollinstanz über die nationalen Glühlampen-Märkte und ihre jeweilige Entwicklung im weltweiten Gewerbe. Andererseits etablierte Phoebus einige weitreichende und langlebige Standards, etwa den bis heute üblichen Glühlampen-Sockel E27. Der zentrale Punkt von Phoebus' Bestrebungen galt jedoch der Glühlampe selbst, und zwar ihrer Lebensdauer bzw. deren Beschränkung. Bereits im folgenden Jahr 1925 wurde diese von einem eigens gebildeten Exekutivkommittee auf nunmehr 1.000 Stunden festgeschrieben, um mit der alsdann reduzierten Brenndauer höhere Verkaufszahlen zu gewinnen. In der oben benannten Literatur zur Frage von Lebensdauern und Verschleiß gilt dies als das technik- wie wirtschaftshistorisch erste Auftreten einer geplanten Obsoleszenz: Unternehmerseitig wurden hier Lebensdauern festgelegt, die unterhalb des technisch-materiell möglichen Optimums lagen. Das Glühlampen-Beispiel ragt dabei insofern hervor, weil es darin um Life-cycle-Bestimmungen abseits modischer Erwägungen geht; in anderen Marktbereichen werden demgegenüber Modewechsel als Begründung für kurze Lebensdauern zentral. Das Beispiel wirkt jedenfalls schulbil-

8 William Meinhardt, Entwicklung und Aufbau der Glühlampenindustrie, Berlin 1932, S. 106, der fast eine Dekade nach Gründung des Kartells damit eine kleine Monografie zur Entstehung und Entwicklung von Phoebus publizierte, das maßgeblich auf ihn selbst zurückgeht. Diese Veröffentlichung ist insofern bemerkenswert und entzieht jeglicher verschwörungstheoretischen Einordnung ihre Grundlage, weil sie jeder Verheimlichung vorzubeugen scheint, wird doch hier über Phoebus offen berichtet. Die vermeintliche Transparenz stößt jedoch schnell an ihre Grenzen, insofern von den technischen Maßnahmen zur Lebensdauerreduktion und dem ,1000h-life-comittee' keine Rede ist.

9 Zu Phoebus vgl. Mária Hidvégi, Internationale Kartelle und der europäische Wirtschaftsraum der Zwischenkriegszeit, in: Themenportal Europäische Geschichte, 2011; online unter: http://www.europa.clio-online.de/2011/Article=482; aus wirtschaftshistorischer Sicht und die enge Kooperation mit GE betreffend vgl. Leonard S. Reich, Lighting the Path to Profit. GE's Control of the Electric Lamp Industry, 1892-1941, in: The Business History Review 66, 1992, S. 305-334, hier S. 324-327. 
dend, sowohl in anderen Sparten der Elektroindustrie, etwa für sogenannte Weißware, als auch in anderen Branchen, etwa bei dem Automobilhersteller General Motors, und für deren jeweilige Gegner. Denn einen Kampf führt Phoebus nicht nur anfangs gegen technische Schwierigkeiten, die Lebenszeit der Lampen erfolgreich von den zuvor üblichen 1.500 bis 2.000 Stunden auf den neuen Wert zu vermindern, sondern auch vehement gegen unfügsame eigene Mitglieder und immer auch gegen vereinzelte Kartellgegner. ${ }^{10}$ Von deren Versuchen, die Brenndauer ungeachtet der wachsamen Phoebus-Augen in alte Höhen oder die Preise in die Tiefe zu schrauben, wird immer wieder berichtet. Und ebenso oft von ihrem Scheitern. ${ }^{11}$

Wie wirksam diese dunklen Machenschaften gewesen sind, lässt sich heute, am Ende der Epoche der Glühlampe, umso klarer bilanzieren. Denn die künstlich begrenzte Lebensdauer ist bei den bis September 2012 noch EUweit produzierten Glühlampen weiterhin wirksam. Mehr noch, eingedenk der inzwischen gesteigerten Netzspannung erwarten handelsübliche Glühlampen derzeit bereits nach ca. 750 Stunden ihren werkseitig eingebauten Brennschluss. 750 Stunden Brenndauer entsprechen fast genau einem Monat. Das lässt sich vergleichsweise gut protokollieren. Ob die nunmehr zu verwendenden, ungleich teureren Energiesparlampen allerdings tatsächlich ihre 20.000 Stunden lang - etwas mehr als zwei Jahre - Licht spenden, wird kaum ein Hausmeister oder Wohnungsbesitzer noch nachprüfen. Bevor nun genauer untersucht sei, wie der Fadentod der Glühlampe geplant und administriert worden ist, sei die Genese des Wirtschaftskonzepts einer geplanten Obsoleszenz kurz umrissen.

\section{Zur Planung in der „Planned Obsolescence“}

Alles unterliegt dem zweiten Hauptsatz der Thermodynamik. Alle Gegenstände verfallen mit der Zeit, weil sie der Entropie ausgesetzt sind. Vom Prozess der Veralterung sind auch technische Gegenstände nicht ausgenommen, weshalb man hier - ganz ohne der Gefahr einer Bio- oder gar einer Anthropomorphisierung zu erliegen - überhaupt von Lebensdauern der Objekte sprechen kann. Das hat als Idee auch Eingang in kultur- und technikhistorische Artefaktgeschichten gefunden, die von „Dingbiografien“" sprechen und den „Lebenszyklus“ der Objekte von ihrer Produktion über den Gebrauch bis hin zum Reparieren und auch zur Dysfunktionalität und zum Entsorgen betrachten, selbst wenn die konkreten Lebensdauern darin selten betrachtet werden. ${ }^{12}$ Ganz allgemein lässt

10 Vgl. dazu Krajewski (wie Anm. 7).

11 Vgl. dazu etwa die zeitweilige Bedrohung im schwedischen Markt durch die Kooperative Förbundet, deren Geschäftsgebaren 1932 von Phoebus genauestens beobachtet wurde, bevor geeignete Gegenmaßnahmen, etwa in Form von einzuschränkenden Drahtlieferungen oder Patentschlachten, ergriffen wurden, vgl. Berliner Landesarchiv A, Phoebus/Osram-Akten, K 683, B1. 183-186, Korrespondenz der Stockholmer Zweigstelle von Osram mit der Hauptgeschäftsstelle in Berlin im März 1932.

12 Vgl. etwa Steven D. Lubar u. W. David Kingery (Hg.), History from Things. Essay on Material Culture, Washington 1993; oder detailliert im Sinne einer Produktlinienanalyse Sudrow 
sich Obsoleszenz bei industriell gefertigten Produkten, in Anlehnung an die maßgebliche Einteilung von Vance Packard (1960/1961), in vier Varianten unterscheiden:

- der gleichsam natürliche Verschleiß durch händische, maschinelle Abnutzung oder schlicht durch Entropie, eine Art technische ,Biodegradation

- die Konkurrenz durch verbesserte Produkte, kurzum, der Effekt, den man als Segnung des technischen Fortschritts verbuchen kann, also z.B. die Ersetzung der Handkurbel am Automotor durch einen elektrischen Anlasser ab 1913 (,funktionelle Obsoleszenz"“13)

- modische Obsoleszenz, auch ,neomania ${ }^{\text {'14 }}$ genannt, also das Verlangen der Konsumenten, ihr vollkommen funktionsfähiges Produkt durch ein nahezu baugleiches, vermeintlich verbessertes Erzeugnis zu ersetzen, à la „der Heckspoiler des neuen Z4 sieht einfach so mega-geil aus, ich konnte nicht widerstehen, hab's gleich gekauft“ (,modische Obsoleszenz“ bzw. „psychologische Obsoleszenz"15)

- die verbraucherunfreiwillige Variante, das heißt der werkseitig vorbestimmte, eingebaute Verfall des Objekts durch verborgene Sollbruchstellen (,qualitative Obsoleszenz"16).

Nur die letzten drei, also ein Austausch durch technisch eindeutig verbesserte Produkte, eine freiwillige Substitution seitens der Konsumenten nach modischen Gesichtspunkten sowie die werkseitig eingebauten Sollbruchstellen werden in der Literatur üblicherweise unter der Rubrik, geplante Obsoleszenz gefasst. Denn sie ziehen eine intendierte Ersetzung nach sich, die einem bestimmten ökonomischen Kalkül unterliegt. Dabei sind die Übergänge zwischen den einzelnen Spielarten fließend und im Einzelfall kaum zu ziehen: Modische und funktionelle Obsoleszenz treffen beispielsweise in der Waschmaschine der 1970er Jahre zusammen, die bei $100{ }^{\circ} \mathrm{C}$ waschen kann (etwa die im VEB Waschgerätewerk Schwarzenberg gefertigte WM 66) und die alsbald von Geräten mit digitalen Bedienschaltflächen abgelöst wird; die qualitative Obsoleszenz wiederum bleibt für Verbraucher gänzlich unsichtbar und allerhöchstens an Garantiezeiten erahnbar. Wesentlich für alle Spielarten der ,geplanten Obsoleszenz' bleibt dabei, dass sie sich prinzipiell einer leichten Reparierbarkeit

(wie Anm. 5), insb. S. 34ff.; von Biografien der Dinge sprach zuerst Arjun Appadurai, The Social Life of Things: Commodities in Cultural Perspective, Cambridge 1986.

13 Vance Packard, Die große Verschwendung. The Waste Makers, Düsseldorf 1960/1961, S. 73.

14 "The insatiable desire to acquire the newest goods in a process of continuous and conspicuous consumption"; Prasad Boradkar, Designing Things. A Critical Introduction to the Culture of Objects, Berg u. Oxford 2010; online unter: www.designingthings.org.

15 ,Modisch“ bei Packard (wie Anm. 13), S. 73; Bodenstein/Leuer sprechen von ,psychologisch', vgl. Gerhard Bodenstein u. Hans Leuer, Obsoleszenz - ein Synonym für die Konsumgüterproduktion in entfalteten Marktwirtschaften, in: Zeitschrift für Verbraucherpolitik/Journal of Consumer Policy 5, S. 39-50.

16 Packard (wie Anm. 13), S. 73. 
der technischen Objekte widersetzen. Die Geräte werden zunehmend als Blackbox organisiert, die für den Konsumenten nicht (leicht) zu öffnen sein soll. ${ }^{17}$ Zalles-Reiber fügt diesen Unterscheidungen weitere Kategorien hinzu, die allerdings eher als Veralterung zu werten sind und nicht unter die Rubrik des gezielten Verschleißes fallen: die ökologische Veralterung, wenn Produkte aussortiert werden, weil ihre Umweltkosten höher als bei neueren Alternativen liegen, und eine ökonomische Veralterung, wenn ein vergleichbares Produkt ein besseres Preis-Leistungsverhältnis bietet. ${ }^{18}$

Die wissenschafts- wie technikgeschichtlich interessanteste Spielart des geplanten Verschleißes ist freilich die letztgenannte Variante, die qualitative Obsoleszenz durch verborgene Sollbruchstellen, und zwar schon deshalb, weil es hier letztlich (wieder) um ein epistemisches Ding geht. Unter einem epistemischen Ding lässt sich nach Hans-Jörg Rheinberger ein zunehmend interessanter werdendes Objekt im Verlauf eines prinzipiell offenen wissenschaftlichen Erkenntnisprozesses verstehen. Dieses Ding zieht innerhalb eines Experimentalsystems im Vergleich zu anderen am Experiment beteiligten Objekten mehr Fragen auf sich und gerät damit allmählich zum Zentrum des gesamten Experiments; das Forschungsinteresse gruppiert sich also vornehmlich um jenes epistemisch prominente Ding, das eine besondere Aufmerksamkeit auf sich zieht, allein weil es neue und andere Fragen hervorbringt als die üblichen, stabilen, weniger hinterfragbaren Elemente des Experiments. ${ }^{19} \mathrm{Im}$ Folgenden wird es darum gehen, diese Denkfigur des epistemischen Dings im Schein der Glühlampe und ihrer experimentell zu begrenzenden Lebensdauer etwas genauer in den Blick zu nehmen. Denn bemerkenswert am Konzept der geplanten Obsoleszenz ist der Umstand, dass das Objekt im Verlauf von Phoebus' Einflussnahme entgegen der üblichen Fortschrittsentwicklung nicht auf technisch-wissenschaftliche Verbesserung seiner Leistungsdaten ausgerichtet ist, sondern - im Gegenteil - die gezielte Implementierung eines Verfalls angestrebt wird, und eine an sich ,produktionsreife" Technik bzw. mit Rheinberger

17 Auch dieser Trend kommt freilich nicht ohne einen Gegentrend aus, in diesem Fall die Tinkerer-Bewegung, die sich inzwischen in Form von Reparatur-Cafés urban organisiert hat, wo man auf die Spezialschraubenzieher für Eingriffe in ein iPhone ebenso zurückgreifen kann wie auf eine umfassende Expertise in Sachen Elektronik-Bastelei. Zu dieser neuen Kultur des Reparierens vgl. z.B. Wolfgang M. Heck1, Die Kultur der Reparatur, München 2013, S. 42-49, der sich auch explizit gegen den Trend zur geplanten Obsoleszenz auspricht. Auf die noch vergleichsweise großen Interventionsmöglichkeiten in der Frühphase der Glühbirnen-Entwicklung, als eine ganze Kleinindustrie von der Reparatur von Kohlefäden lebte, weist Luxbacher (wie Anm. 4) hin.

18 Vgl. Manuel Zalles-Reiber, Produktveralterung und Industrie Design, München 1996, S. 264.

19 Vgl. Hans-Jörg Rheinberger, Das epistemische Ding und seine technischen Bedingungen, in: ders. (Hg.), Experiment, Differenz, Schrift. Zur Geschichte epistemischer Dinge, Marburg a.d. Lahn 1992, S. 67-86; ders., Experimentalsysteme, Epistemische Dinge, Experimentalkulturen. Zu einer Epistemologie des Experiments, in: Deutsche Zeitschrift für Philosophie 3, 1994, S. 405-417. 
gesprochen: ein technisch stabiles Objekt, wieder ins Labor wandert und zu einem epistemischen Ding wird.

Doch zunächst seien noch ein paar genealogische Sondierungen zur Herkunft des Begriffs gleichermaßen wie zu den Ursprüngen weniger eines epistemologischen als des ökonomischen Konzepts einer geplanten Obsoleszenz gestreift.

Die vermutlich erste explizite diskursive Erwähnung der Begrifflichkeit, die einer praktischen Erprobung im Klandestinen der Glühlampenindustrie um rund ein halbes dutzend Jahre hinterherhinkt, lässt sich mit dem Pamphlet des New Yorker Immobilienmaklers Bernard London auf 1932 datieren. Unter dem Titel Ending the Depression through Planned Obsolescence entwickelt London die Idee eines dezidierten Todeszeitpunkts von Produkten, der bereits in der Fertigung der Erzeugnisse zu bestimmen und entsprechend ingenieurstechnisch einzubauen sei. Bemerkenswert bleibt hier einmal mehr die disziplinäre Durchkreuzung der Diskurse, insofern London gleichsam eine ,juridische' Obsoleszenz imaginiert, die zunächst in Form einer Art staatlicher Wirtschaftsförderung als Krisenphänomen einzuordnen ist, später jedoch sowohl technische Maßnahmen als auch Adaptionen im privatwirtschaftlichen Sektor zur Folge haben kann.

Ausgangspunkt seiner Überlegungen bildet einerseits - der Titel des Pamphlets macht es deutlich - die wirtschaftliche Schieflage der USA infolge des großen Börsencrashs 1929, andererseits der Befund, dass die Krise verschärft wird durch die große Zurückhaltung der Bürger, die wenig amerikanische Produkte kauften und so die Binnennachfrage lähmten. Der Effekt: Die Warenhäuser quellen über und die Wirtschaft darbt, weil es an Käufern mangelt, die ihrerseits allzu lange ihre bereits vorhandenen Güter weiternutzen. Die Lösung sieht London derweil darin, den Dingen ein Verfallsdatum einzubauen, und zwar in alle Produkte, Schuhe, Häuser, Maschinen, direkt bei der Produktion. "Briefly stated, the essence of my plan for accomplishing these much-to-bedesired-ends is to chart the obsolescence of capital and consumption goods at the time of their production." ${ }^{20}$ Nach Ablauf dieses Verfallsdatums verwandeln sich die Dinge in einen Status, den London ,rechtlich tot" nennt. "After the allotted time had expired, these things would be legally 'dead' and would be controlled by the duly appointed governmental agency and destroyed if there is widespread unemployment." ${ }^{21}$ Diese Regierungsagentur zahlt demnach eine nationale, allumfassende Abwrackprämie auf beliebige von ihr kontrollierte Güter. Zugleich soll sie über ein Strafsystem verfügen, indem sie befugt wäre, Steuererhöhungen für jene zu verhängen, die keine neuen Produkte kaufen, sondern es vorziehen, ihre alten und ,nutzlosen' Dinge weiter zu nutzen. ${ }^{22}$

20 Bernhard London, Ending the Depression through Planned Obsolescence, Selbstverlag 1932, S. 2.

21 Ebd.

22 Ebd., S. 3. 
Festgelegt werden die Lebensdauern der Produkte derweil von einer Allianz aus Ingenieuren, Ökonomen, Mathematikern, allesamt Spezialisten in ihrem Feld, im Auftrag der Regierung, wobei sich London darüber ausschweigt, wie genau sowohl die jeweiligen Lebensdauern zu ermitteln als auch die Expertenkommissionen zusammenzusetzen seien. ${ }^{23}$ In dem achtseitigen Pamphlet fällt ein einziger Satz zur Destination der ausgesonderten Objekte: "They should be thrown into a junk pile". ${ }^{24}$

Wenngleich über die Verbreitung dieser Streitschrift, die im Selbstverlag erschien und durchaus Flugblattcharakter besitzt, sich wenig rekonstruieren lässt, tauchen zeitgleich vergleichbare Vorschläge auf, etwa das von Roy Sheldon und Egmont Arens in ihrem einflussreichen Buch Consumer Engineering. A New Technique for Prosperity verfochtene Konzept einer 'progressive obsolescence', wo sie das Phänomen als eine ökonomische Bewegung charakterisieren, die nach dem Ersten Weltkrieg wirtschaftswissenschaftliche Führung erhalten habe, nicht zuletzt inspiriert von tayloristischen Überlegungen. ${ }^{25}$ Als Aufgabe des Consumer Engineering beschreiben sie das Ziel, "that we use up the kind of goods we now merely use" - langlebige Konsumgüter sollen also ebenso „verbraucht" werden wie „Verbrauchsgüter“.

Die ersten praktischen Versuche eines gezielten Herunterschraubens von Nutzungs- und damit Lebenszeiten, die dann allmählich beobachtbar und damit theoretisierbar wurden, gehen einerseits auf die Elektroindustrie im Allgemeinen und die Glühlampenindustrie im Besonderen, andererseits auf die Automobilindustrie zurück, also tatsächlich auf Industrien, die den Taylorismus vorrangig umzusetzen suchten. Als Paradebeispiel der modischen Obsoleszenz gelten die Maßnahmen von Alfred P. Sloan, dem langjährigen Präsidenten und CEO von General Motors, mit denen die Marktmacht des Konkurrenten Henry Ford bekämpft wurden, um sich gegen dessen langlebigen Produkte mit ausdifferenzierten Fahrzeug-Designs durchzusetzen. Als zentral für den Sloanismus gilt eine Umstellung der Produktionszyklen hin zu einem raschen Modellwechsel, der im Gegensatz zum höchst beständigen Modell T von Ford im jährlichen Rhythmus mit kosmetischen oder vorwiegend optischen Veränderungen aufwartete, um potenziellen Käufern mit Hilfe von geringen Modifikationen etwas technisch wenig verändertes als überragende, neue Errungenschaft anzupreisen. ${ }^{26}$

Ob nun Automobile, Nylonstrümpfe, Spanplatten, Waschmaschinen oder Glühlampen, das Prinzip der geplanten Obsoleszenz hat sich vermutlich in

23 Ebd., S. 4.

24 Ebd., S. 6.

25 Vgl. Roy Sheldon u. Egmont Arens, Consumer Engineering; A New Technique for Prosperity, New York 1932, S. 53f., hier S. 13. Vgl. auch die Einleitung zu diesem Sonderheft.

26 Giles Slade, Made to Break. Technology and Obsolescence in Amerika, Cambridge/Mass. 2006, S. 29-56. 
zahlreichen Industriezweigen fundamental verankert. ${ }^{27}$ Ähnlich wie London es vorschwebte, sind wir umgeben von Produkten mit überschaubaren Lebensdauern, die meist gerade eben über die gesetzlich vorgeschriebene Mindesthaltbarkeit - sprich: Garantiefrist - hinausreichen. Eine staatlich orchestrierte Aktion wie die sogenannte „Abwrackprämie“ deutet sogar darauf hin, dass das Verkürzen von Lebensdauern technischer Artefakte sich als akzeptierte Strategie durchgesetzt hat, um die Wirtschaft zumindest in vermeintlichen Krisen anzukurbeln. Gleichzeitig beginnen design- und ingenieurwissenschaftliche Abhandlungen zu einer „grünen“ Produktgestaltung soeben, die Planung der Lebensdauern der Dinge im Massenkonsum kritisch zu reflektieren. ${ }^{28}$ Verbraucherschützer fordern verbindliche Angaben der Hersteller zu den geplanten Lebensdauern ihrer Geräte. Den Unmut der Nutzer über inzwischen von ihnen eindeutig als zu kurz empfundene Lebenszeiten hat kürzlich der ARTE-Film Kaufen für die Müllhalde von Cosima Dannoritzer (2010) eingefangen. Der Film hat damit die populäre Debatte um den geplanten Verschleiß wiederbelebt, wie sie ähnlich bereits in den 1970er Jahren - angeregt von Packard, entzündet von Ingo Schmitt (1971), entfacht hingegen durch ein von der damaligen Bundesregierung in Auftrag gegebenes Gutachten des Volkswirtschaftlers Burkhardt Röper (1976) - in der deutschsprachigen Öffentlichkeit zu Kontroversen geführt hatte..$^{29} 1982$ legte ob der Wogen der Debatte auch die OECD eine Studie zu der Frage des geplanten Verschleißes vor. Sie stellte den Auswuchs von nichtreparierbaren sowie unmittelbar für das Wegwerfen produzierten Dingen heraus und betonte einerseits, dass sich zwar keinerlei gezielte Lebensdauerverkürzung nachweisen ließe, dass Unternehmen anderer-

27 Vgl. Boradkar (wie Anm. 14).

28 Vgl. Timothy Cooper, Longer Lasting Products: Alternatives to the Throwaway Society, Farnham 2010; Stuart Walker, Jacques Giard u. Helen L. Walker, The Handbook of Design for Sustainability, London u. New York 2013.

29 Vgl. für den deutschsprachigen Raum die Debatte in den 1970er Jahren, ausgehend von Ingo Schmidt, Obsoleszenz und Mißbrauch wirtschaftlicher Macht, in: Wirtschaft und Wettbewerb 21, 1971, S. 868-877; Jörg Glombowski, Ansätze zu einer Theorie der geplanten Obsoleszenz, Berlin 1976; vor allem aber das Gutachten von Burkhardt Röper, Gibt es geplanten Verschleiß? Untersuchungen zur Obsoleszenzthese, Göttingen 1976; und die daran anschließende, zum Teil heftige Debatte u.a. von Karl-Heinz Hillmann, Geplante Obsoleszenz. Bemerkungen zu Burkhardt Röper: Gibt es geplanten Verschleiß?, in: Zeitschrift für Verbraucherpolitik/Journal of Consumer Policy 1, 1977, S. 48-61; Burkhardt Röper, Gibt es geplanten Verschleiß? Eine Antwort auf die Kritik von Hillmann in ZVP, 1, 1977, in: Zeitschrift für Policy 1, 1977, S. 185-194; Gerhard Bodenstein u. Hans Leuer, Geplanter Verschleiß in der Marktwirtschaft, Frankfurt a.M. u. Zürich 1977; Bodenstein/Leuer (wie Anm. 15); Hans Raffée u. Klaus-Peter Wiedmann, Obsoleszenz - eine deklaratorische Kategorie? Stellungnahme zum Beitrag von Bodenstein und Leuer in ZVP, 5, 1981, in: Zeitschrift für Verbraucherpolitik/Journal of Consumer Policy 5, 1981, S. 357-365. Zur jüngeren Debatte vgl. auch Jürgen Reuß u. Cosima Dannoritzer, Kaufen für die Müllhalde. Das Prinzip der geplanten Obsoleszenz, Freiburg 2013 sowie Robert Friedell, Obsolescence: Origins and Outrages. Cosima Dannoritzer, „The Light Bulb Conspiracy“, in: Technology \& Culture 54, 2013, S. 167-169. 
seits aber Lebensdauern verlängernde Innovationen durchaus zu unterdrücken schienen, wie es das Beispiel der Glühbirne zeige. ${ }^{30}$

Wie aber könnte sich, wenn überhaupt, die technische Umsetzung des Marketingideals eines exakt implementierten Verschleißes recherchieren, analysieren und beschreiben lassen? Jedem Produkt ist ein Interessenkonflikt inhärent, ein Konflikt zwischen gebotener Langlebigkeit, Kostenkalkulation und möglicher Fertigungsqualität des Objekts, zwischen einer Präsentation seiner hervorzuhebenden Eigenschaften durch die Hersteller und einem Verbergen der für den Verbraucher problematischen. Und es ist gerade die Opazität seitens der Hersteller, die nicht nur dem Kunden den Blick in die Produktionsstrategien und -stätten verwehrt, sondern mehr noch für den Historiographen technischer Systeme ein Problem darstellt. Darin liegt die fundamentale Schwierigkeit insbesondere für historische Studien zum Thema von Lebensdauern technischer Objekte und insbesondere der geplanten Obsoleszenz: Das Quellenmaterial ist eigentlich nicht existent, weil jede Produktionsabteilung darum besorgt sein muss, keine - zumal rechtlich relevanten - Spuren zu hinterlassen, die dem Verbraucher Indizien geben könnten, dass der Hersteller seine Geräte gegen das Vertrauen auf und das Interesse der Kunden an Zuverlässigkeit und Langlebigkeit fertigt. Aus diesem Grund muss man in den überwiegenden Fällen die Verhandlungen über etwaige einzubauende Schwach- und Bruchstellen als orale, undokumentierte Kommunikation annehmen, die auf schriftliche Spuren in Form von Geschäftskorrespondenz oder kommentierte Schaltpläne tunlichst verzichtet.

Es ist diese Grundproblematik, die das Thema nicht selten, wenngleich unberechtigt in die Nische von technischen, wissenschaftlichen oder auch historischen Verschwörungstheorien rückt. Dass diese Zuschreibung und die geplante Obsoleszenz im Gegenteil ernsthafte technikwissenschaftliche Forschung erfordert, lässt sich dagegen im Fall von Phoebus - genauer: anhand der Osram-Geschäftskorrespondenz aus den Jahren 1924 bis 1941 - exemplarisch vorführen. Allerdings darf man dabei nicht vergessen, dass es sich um Briefe von Verwaltungsdirektionen untereinander handelt, die allenfalls beiläufig das Geschehen in ihren Entwicklungslaboren dokumentieren und auf diese Weise eher indirekt Einblick gewähren in die technischen Abläufe, mit denen geplante Obsoleszenz umzusetzen ist.

\section{Phoebus' Projekt: Versuchsreihen zur Reduktion der Lebensdauer}

Wenn die Direktoren der führenden Glühlampenhersteller am Heiligabend 1924 im Zeichen von Phoebus nicht nur beschließen, den Weltmarkt für Leuchtkörper unter sich aufzuteilen, sondern zugleich noch eine Reduktion der Lebensdauer für die einzelnen Lampen in Aussicht stellen, dann ist

30 Vgl. OECD, Product Durability and Product-Life Extension: Their Contribution to Solid Waste Management, Paris 1982. 
damit zunächst einmal ein Projekt auf dem Papier beschlossen, das in den Folgejahren (bis in die 1930er Jahre) in Form mühsamer technischer Experimentierreihen realisiert wird. An diesem Projekt lässt sich die Interferenz von neuen ökonomischen Plänen und der technisch exekutierenden Forschungsund Entwicklungsabteilungen ermessen, die unter tunlichster Geheimhaltung mit wissenschaftlichen Mitteln wirtschaftliche Vorgaben zu befolgen hat: die „[t]echnische Rationalität gehorchte [...] der ökonomischen Rationalität" und sie wurde in das Produkt auf eine für den Konsumenten unsichtbare Weise eingeschrieben. ${ }^{31}$

Neben dem übergeordneten Verwaltungsrat besitzt Phoebus vier exekutive Abteilungen: für den Verkauf, die Preis-Politik, die Propaganda und schließlich die Technik. Die Abteilung für Verkauf betreut nicht etwa weltweit die Kunden aller Phoebus angehörigen Firmen, sondern wacht ausschließlich über die Verkaufsbilanzen der Kartellmitglieder. Um einen geordneten Verkauf innerhalb eines wettbewerbsfreien Weltmarktes zu gewährleisten, teilt Phoebus den Markt in drei verschiedene Segmente bzw. Typen ein: Zum Beispiel verfügt Tungsram über Ungarn als heimischen Markt, der von der Firma konkurrenzlos kontrolliert wird. Ihr Kontingent auf dem europäischen Markt liegt bei ca. 25\% und im übrigen Teil der Welt darf Tungsram zu geringeren, gestreuten Anteilen Glühlampen verkaufen. Denn die Anteile innerhalb des gemeinsamen Marktsegments werden nach vormaliger Geschäftsmacht vergeben. Als Grundlage dienen die jeweiligen Handelsbilanzen und Glühbirnenabsatzzahlen innerhalb der Jahresdauer vom 1. Juli 1922 zum 30. Juni 1923.32 Die daraus resultierenden Kontingente dürfen im Laufe eines Geschäftsjahres nicht überschritten werden, anderenfalls greifen Konventionalstrafen, die an die Übervorteilten zu entrichten sind. Ein Fond, in dem alle Mitglieder Sicherheiten hinterlassen haben, garantiert die Zahlung der Strafgelder.

Im Glühlampenvertrag wird zwischen den einzelnen Gesellschaften uneingeschränkter Patentaustausch und vorbehaltloser Wissenstransfer festgeschrieben, gefördert und koordiniert von Phoebus' technischer Abteilung. Gleich uneingeladenen Verwandten besuchen Delegationen der Vertragspartner die Forschungs- und Entwicklungslabors ihrer einstigen Konkurrenten, um sich über neue Fertigungstechniken und (marginale) Innovationen zu informieren und nicht zuletzt um die Produktionsverfahren aufeinander abzustimmen. Das Ziel heißt nicht nur Ausgleich von Wettbewerbsvorteilen, sondern vor allem Standardisierung der Produkte. ${ }^{33}$ So präsentiert die zusammengeschlossene Glühlampenindustrie bereits 1926 der Welt einen einheitlichen, an Edisons Typen orientierten Sockel und die zugehörige Fassung, was die Voraussetzung

31 Luxbacher (wie Anm. 4), S. 118.

32 Stocking/Watkins (wie Anm. 7), S. 333.

33 Vgl. auch den Vertrag bei Fritz Werr, Internationale Wirtschaftszusammenschlüsse (Kartell und Konzern) und Staat als Vertragspartner, Berlin 1936, S. 29 und weiterhin Gottfried Eissfeldt, Die Kartellierung der deutschen Elektroindustrie, Berlin 1928, S. 75. 
für ein weltweit greifendes Plug-and-Play darstellt. Immerhin in diesem Punkt lässt sich ein unzweifelhafter Vorteil für den Verbraucher ausmachen: Der E27- bzw. E14-Sockel (,E` steht für Edison, die numerische Angabe für den Durchmesser in Millimetern) ist bis heute gültig und kann als schulbildend für die Durchsetzung von globalen Standards angesehen werden.

Doch nicht nur der Sockel, sondern ebenso einheitliche Richtlinien zur Qualität der Glühlampen markieren ein Politikum des Kartells. Oder im produktionstechnischen Klartext, der den ,Vorteil des Verbrauchers' mit einer Zahl belegt: "The average life of lamps for General Lighting Service must not to be guaranteed, published or offered for another value than 1,000 hours to burn out on normal laboratory test conditions at rated voltage." ${ }^{34}$ Das Standardisierungskommittee befasst sich vor allem mit den Schwierigkeiten, die die Eingrenzung der Glühlampenlebensdauer mit sich bringt. Bereits 1925 wird eine spezielle Arbeitsgruppe, das 1.000 Hour Life Commitee, gegründet, die sowohl die ökonomischen wie die technischen Möglichkeiten eruiert, das Leben der Lampen auf einen Punkt, das heißt den kalkulierten Brennschluss zu bringen. Neben dem Erlass, ab sofort in den firmeneigenen Propagandaabteilungen nicht mehr - wie zuvor üblich - mit Hinweisen auf lange Lebensdauern zu werben, und sie schon gar nicht auf den Glühbirnen zu vermerken, wird eine zweifache Prüfinstanz etabliert, um zu gewährleisten, dass alle dem Kartell zugehörigen Firmen der Direktive nachkommen. Jede Fabrik muss demnach im eigenen Labor die Lebensdauer bestimmen, die Werte nebst zufällig ausgewählten Birnen an Phoebus senden, wo die Lampen erneut auf ihre unerwünschte Langlebigkeit hin untersucht werden.

Die Koordinatoren des weltweiten Glühlampengeschäfts sehen die Problematik der 1.000-Stunden-Vorgabe in zweierlei - in technischer wie in ökonomischer - Hinsicht, aber es sind in ihren internen Dokumenten vorzugsweise ökonomische Gründe, die für die Nivellierung und Reduzierung der Lebensdauern sprechen: "From a commercial point of view it is of very great importance to surpass the burning-life of 1.000 hours as little as possible, as every surpassing of only 10 hours means a loss on the world-contingent of $+/-1 \%$ or about 4.000 .000 units. Technically it is possible to reach an average life of 1.000 hours to a few percentages exact, when manufacturing very carefully." ${ }^{35}$ Die sorgfältige Fabrikation der Glühlampen beschäftigt die Ingenieure und Standardisierer jedoch weit weniger als die Frage, welches

34 Berliner Landesarchiv A, Phoebus/Osram-Akten, K 1158: Interne Korrespondenz des Standardizing Committee der Phoebus S.A., Resolution on The Enforcement of the Standard Life for General Lighting Service Lamps, Brüssel, im April 1929; Berliner Landesarchiv A, Rep. 231, Nr. 0.122: Brief von C.A. Atherton an andere Phoebus-Vorstände vom 24.3.1936. Festgelegt wurde die Dauer eines jeden Birnenlebens am 17./18. Februar 1925 auf einer Tagung des General Committees in Paris, vgl. Berliner Landesarchiv A, Phoebus/OsramAkten, K 693, A Rep. 231, Nr. 0.76.

35 Nach einer Eingabe der Philips Glowlamp Works, Ltd., Eindhoven Holland vom 7.3.1928, vgl. Berliner Landesarchiv A, Phoebus/Osram-Akten, A Rep. 231, Nr. 97. 
technische Verfahren angewendet bzw. nach welcher Formel die Lebensdauer der zu prüfenden Zufallsbirnen bestimmt werden soll. Während die technischen Stellgrößen für die zu begrenzende Lebensdauer sich vor allem auf den Draht und seine Befestigung konzentrieren - hier sind etwa die Spritzverfahren des Wolframmaterials, seine genaue Zusammensetzung, die Homogenität des Fadens als jene Elemente, an deren Modifikation sich mit der Lebensdauer experimentieren lässt, zu nennen -, stellen die statistischen Methoden, nach denen in den einzelnen Fabriken die Lebensdauern bemessen werden, eine noch auszuhandelnde unbekannte Größe dar. Nur folgerichtig entbrennt ein phoebusinterner Streit, der eine neue, abgeschirmte Forschungsdiskussion mit geheimen „Veröffentlichungen" sowohl technischer als auch stochastischer Fragen entstehen lässt. ${ }^{36}$ Die Beiträge zirkulieren in einem regen postalischen Wechsel zwischen den einzelnen Labors samt Durchschlägen für die Vorstandsetagen, um die unterschiedlichen Berechnungsverfahren in eine Formel zu bemühen. Zum Beispiel mit der Lebensdauerberechnung nach $\mathrm{C}$ (= Commercial figure of merit)

$$
C=\frac{L_{m}}{L_{0}} \cdot 100-F-\frac{1}{10}(100-U)
$$

(mit $\mathrm{L}_{\mathrm{m}}$ als mittlerer Lebensdauer der Lampen, $\mathrm{L}_{0}$ als Standardlebensdauer, F als Zahl der Frühdurchbrenner und U als Gleichförmigkeitsfaktor der Anfangsmessung).

Schnelle Einigung erzielt man - im Gegensatz zur langandauernden Kontroverse um die vermeintlich wahre Bestimmung der Lebensdauer, die sich als ein komplexes Produkt aus fertigungstechnischen Details ergibt, ökonomisch hingegen der Effekt einer diskursiven Übereinkunft unterschiedlicher Vorstellungen der beteiligten Firmen bleibt - jedoch nur in der Übereinkunft, die Lichtleistung fortan, statt der vorher üblichen Angabe in Kerzenstärke, nunmehr in Watt zu bemessen.

Nachdem man sich in Fragen nach der Lebensdauerformel hat einigen können, wird die 1.000-Stunden-Problematik aus ökonomischer Perspektive gelöst, indem linear gestaffelte Konventionalstrafen eingeführt werden; eine zuverlässige Eruierung der Lebensdauern ist also auch deshalb nötig geworden, um solche Strafen korrekt zu verteilen: ${ }^{37}$

36 Vgl. etwa die Typoskripte von Hubert Curt Plaut, „Wirkung von Wechselstrom nicht sinusförmigen Verlaufs auf die Dauer von Glühlampen“" oder aber Marcello Stefano Pirani, „Die Wahrscheinlichkeit von Zufallsfehlern in den Lebensdauerbestimmungen“. Nach einiger Latenzzeit lüftet man den Schleier, um die internen Forschungsergebnisse in den hauseigenen Organen, vor allem in den „Technisch-Wissenschaftlichen Abhandlungen aus dem Osram-Konzern“, 1930ff., zu veröffentlichen.

37 II. General Board Meeting of the G.P. \& B.D.A. of the S.A. Phoebus, Paris March 28/291928; Resolution on The Enforcement of the Standard Life for General Lighting Service Lamps, S. 4f. 
„(2) Any Party of the agreement, the average life of whose General Lighting Service Lamps of the voltage and wattage ratings indicated in clause (1), exceeds 1750 hours or is less then 800 hours during the twelve months ending 30th June 1929 , or exceeds 1500 hours or is less then 800 hours during each subsequent Fiscal Period, shall pay fines as indicated in clause (4). [...]

(4) The fine shall be calculated according to the following scale:

Fines for the 4th Fiscal Period

Average Lamp Life

Fine in Swiss francs per thousands units sold of the ratings mentioned in Clause (1) during the Fiscal Period ending 30th June 1929

$\begin{array}{ll}0-599 \text { hours } & 20 \\ 600-799 \text { hours } & 5 \\ 800-1750 \text { hours } & \text { no fine } \\ \text { 1751-2000 hours } & 20 \\ \text { 2001-2500 hours } & 50 \\ \text { 2501-3000 hours } & 100 \\ \text { over 3000 hours } & 200 “\end{array}$

Diese Vorgaben führen nach zweimaliger Prüfung der Lebensdauern - in den Labors der Hersteller und dann bei Phoebus - beispielsweise in der ,IV. Fiscal Period‘ zu folgenden Strafen, wobei die Abweichler nur anonym in den Statistiken erscheinen: Bei den luftleeren Lampen müssen fünf Parteien, d.h. Gesellschaften jeweils folgende Beträge entrichten: 16.000, 6.000, 118.000, 62.000 und 102.000 SFr, also insgesamt 304.000 SFr, bei gasgefüllten Lampen beträgt die Gesamtsumme der Strafgelder insgesamt 205.000 SFr. In der, V. Fiscal Period" verschieben sich die Beträge nicht nur wegen des geänderten Berechnungsverfahrens zu 156.000 SFr bei den luftleeren resp. $483.000 \mathrm{SFr}$ bei den gasgefüllten Lampen. Die Vakuumbirne verzeichnet demnach scheinbar eine sorgfältigere Fabrikation als das technisch noch nicht so gefestigte Herstellungsverfahren von Birnen mit Gasfüllung. Dennoch weisen die Statistiken signifikant höhere Brenndauern bei luftleeren Lampen auf als bei gasgefüllten, was als Indiz dafür zu verstehen ist, dass der von der Direktion vorgegebene Soll-Wert der Brenndauern tatsächlich kontingent ist. Offenbar kann ein neues Produkt, das noch nicht die vorgegebene Brenndauer erreicht hat - sie also möglicherweise gar unterschreitet -, nur mit hohen Strafzöllen in Richtung der Zielvorgabe getrieben werden. Wiederum große Unterschiede bestehen zwischen den Messungen, die Phoebus durchführt im Vergleich zu den werkseigenen Prüfergebnissen. So verzeichnet der Osram-Konzern im Geschäftsjahr 1927 gemittelt über alle Fabrikationsstätten in Berlin, Augsburg, Drammen, Madrid, Pabjanice, Prag und Winterthur eine gewertete mittlere 
Brenndauer von 1.520 Stunden bei luftleeren und von 1.460 Stunden bei gasgefüllten Lampen. Die Phoebus-Prüfstelle misst hingegen 1.490 Stunden bei den Luftevakuierten und 1.170 Stunden bei den Gasgefüllten, was wiederum als Indiz für die seinerzeit bei Phoebus vehement geführte Diskussion gewertet werden kann, wie die Brenndauer überhaupt zu ermitteln sei und welche Formeln dafür Anwendung finden sollten.

Auch unter anderen Prämissen analysieren die Standardisierer in Phoebus' Laboren ihre aufwendigen Messreihen. Es gilt, die Synchronisation von zwei alltäglichen Verbrauchsgütern voranzutreiben: die Lebensdauer der Glühlampe soll der durchschnittlichen Betriebsdauer für Batterien - etwa von Taschenlampen - angeglichen werden, damit die Auffrischungszyklen beider Komponenten fortan zusammenfallen. ${ }^{38}$

Wie kann eine Firma bei festgelegtem Marktanteil dennoch hohe Profite erzielen? Allein verringerte Produktionskosten treiben die Gewinne nach oben. Aus diesem Grund fixiert Phoebus die jeweiligen Preise pro Glühlampe auf relativ hohem Niveau, um die Gewinnspanne erträglich zu halten, also nach kapitalistischem Prinzip: Man zielt darauf, sie zu erweitern, ohne die Rationalisierungsgewinne aber an die Konsumenten weiterzugeben. Obschon keine einheitliche Regulierung der Preise festgesetzt ist, koordiniert die Preisabteilung die Entwicklung durch spezielle länderspezifische Kommissionen, zusammengesetzt aus Vertretern der am Ländermarkt interessierten Produzenten. ${ }^{39}$

Ein Bild (Abb. 1) mag - mehr als tausend Zahlen - jenen Fakt veranschaulichen, dass Phoebus' Plan, dank seiner überaus akribischen und generalstabsmäßigen Projektierung, ein voller Erfolg geworden ist. Zu sehen sind die erhobenen Lebensdauern von Glühlampen - berechnet nach einer allgemein akzeptierten Formel, etwa wie in Formel 1 - über einen zeitlichen Verlauf von neun Jahren, innerhalb dessen sich die Linie der Fabriken, die durchschnittlich höhere oder geringere Lebensdauern produzieren, immer stärker dem vorgegebenen Korridor von 800 bis 1.500 Stunden annähern. Bemerkenswert ist dabei nicht zuletzt, dass ab dem Fiskaljahr 1932/33 keine Fabrik seinen Lampen mehr als 1.500 Stunden gönnt. Dass die Glühlampen-Lebensdauer immer weniger streut, liegt einerseits in Teilen auch an der abschließenden Maschinisierung des Fertigungsprozesses, welche in den Jahren um 1930 die letzten Flaschenhälse der Handarbeit beseitigt; andererseits hätte dies aber keinesfalls mit einer Nivellierung der Lebensdauern hin zu dem niedrigen Wert von 1.000 Stunden verbunden werden müssen. Dies zeigt umgekehrt, dass bereits die teil-manuelle Herstellung der Lampen vor 1934 den PhoebusVorgaben erstaunlich gut genügten.

38 Stocking/Watkins (wie Anm. 7), S. 354.

39 Vgl. Eissfeldt (wie Anm. 33), S. 76; Stocking/Watkins (wie Anm. 7), S. 337 u. 343f; Luxbacher (wie Anm. 5) schätzt für die 1920er Jahre, dass sich die Verkaufspreise knapp halbierten, die Produktivität sich jedoch mehr als vervierfachte, S. 374. 


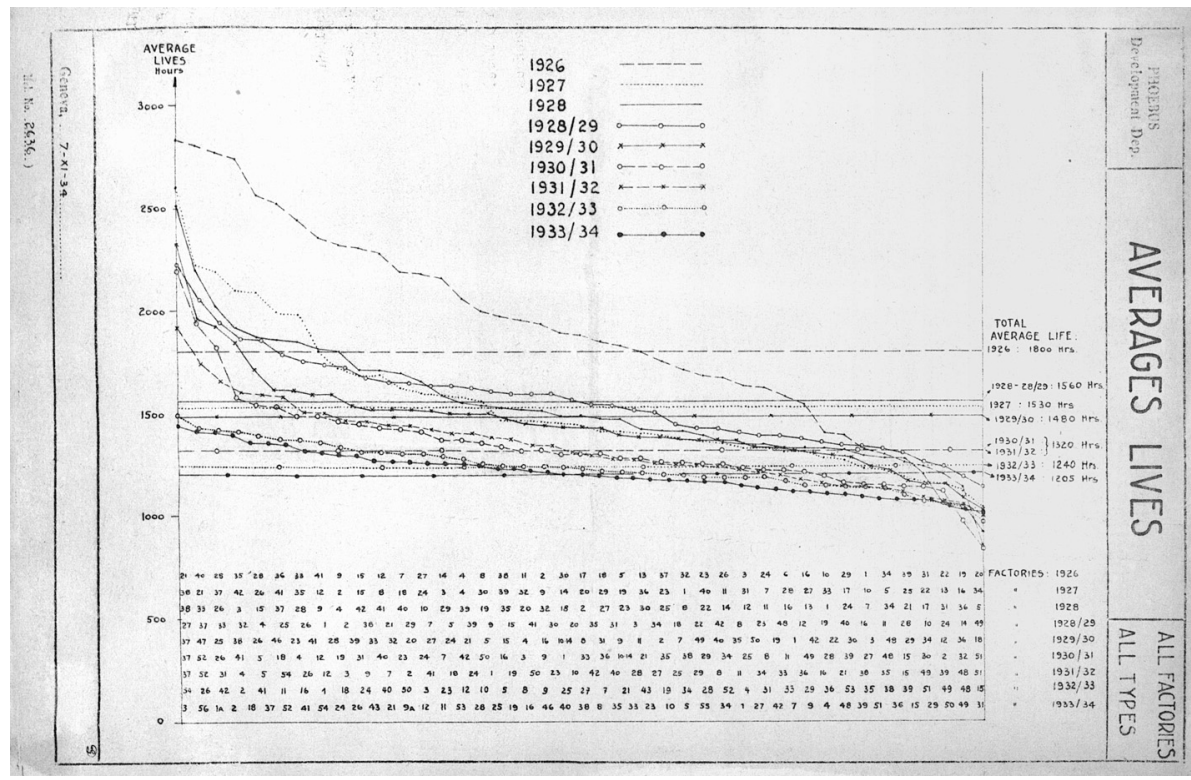

Abb. 1: Übersicht über Lebensdauerangleichungen unterschiedlicher Phoebus-Fabriken innerhalb von neun Geschäftsjahren. Das Diagramm ist nicht unmittelbar leicht zu lesen, denn während die durchschnittlichen Brenndauern auf der y-Achse zu finden sind, zeigt die Abszisse nicht etwa wie üblich die Zeit an. Vielmehr sind dort die unterschiedlichen Fabriken aufgetragen, d.h. die Annäherung an die 1.000-Stunden-Grenze ist als asymptotische Bewegung in der Vertikalen, ein Hinunterwandern der einzelnen Kurvenlinien durch die Geschäftsjahre zu verstehen. Die Wanderungsbewegung der Kurve von einem steilen Abfall hin zu einer möglichst waagerechten Lage dokumentiert die allmähliche Annäherung an die Produktionsvorgabe.

Auch wenn der Glühlampenvertrag, gemäß dem Diktum seines geistigen Vaters, des Osram-Direktors William Meinhardt, allein zum „Vorteil des Verbrauchers" eingerichtet gewesen sei, ${ }^{40}$ so kommt man mit einem nüchternen Blick auf die Organisation und Machenschaften wohl nicht erst heute zu der Bilanz, dass Phoebus zweifelsohne zum ,Nachteil des Verbrauchers' wirkte. Im Kern dieses Arguments steht dabei freilich die Begrenzung der Lebensdauer auf 1.000 Stunden, wie sie im Februar 1925 von Phoebus beschlossen wurde.

Der wissenschaftliche Aspekt dieser Beschlusslage führt derweil zu einer bemerkenswerten Situation, die sich signifikant von den üblichen Erkenntnisstrategien in den Labors, sei es bei Alchimisten oder Biogenetikern, unterscheidet: Das Experimentalsystem orientiert sich hier gerade nicht an den Prämissen eines technischen Fortschrittsgedankens, der seine Bemühungen in den Dienst der Verbesserung der Technik für die Allgemeinheit stellt. Denn wenn die Entwicklungsrichtung im Labor für gewöhnlich vom epistemischen

40 Meinhardt (wie Anm. 8), S. 106. Der Satz bleibt freilich reine Behauptung und wird von Meinhardt argumentativ nicht unterfüttert. 
Ding zum technologischen Objekt führt, ${ }^{41}$ kehrt sich diese Richtung hier um: Die Glühlampe stammt aus dem physikalisch-chemischen Labor der Elektroindustrie und wird dann vom Laborprodukt zu ihrem ersten, industriellen Konsumprodukt, das in Serien- und bald in Massenproduktion hergestellt wird. Um 1900 werden Glühlampen vor allem mit Kohlefäden zum Leuchten gebracht, bevor sich vor dem Ersten Weltkrieg dann die Metallfadenlampe mit ihren Leuchtstoffen auf Basis seltener Erden wie vor allem Wolfram durchsetzt. ${ }^{42}$ Ende der 1920er Jahre kann man die Metallfaden-Glühlampe als technisch weitestgehend ausgereift charakterisieren. Die Lichtausbeute ist beachtlich, die Brenndauern liegen mühelos bei 2.500 Stunden und mehr. Am Objekt gibt es - seitdem nach dem Ersten Weltkrieg die Produktion nahezu komplett maschinisiert erfolgt - eigentlich kaum etwas zu verbessern; den Abschluss der Maschinisierung bildet die Spannmaschine, die zusammen mit der Doppelwendellampe um 1934 eingeführt wurde. Und doch gerät das technologisch ausgereifte Objekt namens Glühlampe wieder zu einem epistemischen Ding, das ein Bündel an Unbestimmtheiten, an offenen Fragen hervorruft, weil die Beschaffenheit des Metallfadens sprichwörtlich - wie das $\mathrm{Rad}$ - neu zu erfinden ist. Es gilt, die zuvor so mühsam angestrebte Langlebigkeit in eine technisch präzis einzustellende Kurzlebigkeit zu überführen. Dazu sind nicht zuletzt aufwendige Experimente und Forschungsanstrengungen, etwa an der Konsistenz oder Geometrie des Wolframdrahts, seiner Pulverspritzgussproduktion notwendig, die von Phoebus in ausgewählten Labors von noch ausgewählteren Wissenschaftlern - allen voran von Prof. Marcello Pirani, dem Direktor der Studiengesellschaft für elektrische Beleuchtung bei Osram in Berlin - unter möglichster Geheimhaltung durchgeführt werden. ${ }^{43}$

\section{The Big Picture}

Möchte man das mit Phoebus, Osram, General Electric, General Motors und anderen Global Playern ab Mitte der 1920er Jahre aufkommende Produktionsparadigma der geplanten Obsoleszenz als eine besonders raffgierige Spielform des Kapitalismus noch in einen weiteren, politisch-ökonomischen Rahmen einordnen, so drängt sich - schon wegen der verwendeten Begriffe - die Planwirtschaft auf: Wie hängt die geplante Obsoleszenz mit der Planwirtschaft zusammen? Die entscheidende Differenz liegt im Folgenden: Das Konzept der geplanten Obsoleszenz generiert einen Defekt und damit einen künstlichen Mangel auf Seiten des Verbrauchers, um diesen durch den

41 Rheinberger (wie Anm. 19), S. 71f.

42 Vgl. dazu auch Krajewski (wie Anm. 7), S. 179-183.

43 Phoebus geht nicht nur aus der Initiative von William Meinhardt (1872-1955) hervor, ihrem Verwaltungsratsvorsitzenden sowie Vorstandsvorsitzenden und treibende Kraft bei der Gründung von Osram als Fusion der Glühlampenzweige von Siemens, AEG und der Auer-Gesellschaft 1919, sondern Osram nimmt innerhalb der Mitglieder zudem eine herausgehobene Stellung ein, was nicht zuletzt durch die tonangebenden Impulse aus den Forschungslabors von Pirani in Berlin untermauert wird. 
Überfluss der Waren seitens des Produzenten zu ersetzen. Die Ersetzungen forcieren die Tendenz, das Benutzbare unbrauchbar zu machen. Das Konzept der Planwirtschaft setzt dagegen auf zuvor entworfene Kontingente an Waren, die über längere Zeiträume, fünf, zehn Jahre ihre Haltbarkeit beweisen müssen. Dass die Planwirtschaft im weiteren Verlauf zu einer Mangelwirtschaft wird, unterstreicht dann zwangsläufig die Notwendigkeit, bei diesem Wirtschaften nicht auf eine Unter- sondern eine Übernutzung des Vorhandenen zu setzen. Dies schließt Reparaturinstanzen ebenso ein wie die systematische Bewirtschaftung von Resten, wie es Möller für die DDR zeigt. ${ }^{44}$

Allerdings hat es auch in Krisenzeiten stets Überlegungen gegeben, das Prinzip der Kurzlebigkeit der Dinge einstweilen zu suspendieren bzw. die sekundären Geschäftszweige wie Reparaturbetriebe und Wiederverwertungsketten zu stärken. So wird im Zug der NS-Kriegswirtschaft etwa die Lebensdauer von Glühlampen auf 1.500 Stunden erhöht, nicht ohne den vehementen Hinweis, in Friedenszeiten sofort wieder zu den bewährten 1.000 Stunden zurückzukehren. ${ }^{45}$ Im Gegensatz zur geplanten Obsoleszenz bestärkt die Wirtschaftsform der Planverfahren also die Tendenz, das Verschlissene zu erhalten und wieder brauchbar zu machen, oder aber gleich so zu fertigen, dass die Objekte außerordentlich langlebig bleiben. ${ }^{46}$

Sofern sich mit einiger Berechtigung - angesichts der permanenten Wirtschaftskrisen - die Frage nach der besseren Alternative stellen lässt, so kann die Antwort zunächst nur eine Gegenfrage zurückgeben: Besser für wen? Für die ,oikonomia', für die Lehre vom ganzen Haus einer Verbraucherfamilie, erweist sich das meist aus dem Mangel geborene Prinzip der Langlebigkeit selbstredend als das günstigere Konzept. Für die Volkswirtschaft ebenso wie für das einzelne Unternehmen verspricht das Konzept der geplanten Obsoleszenz derweil die profitablere Wahl zu sein. Für eine Gesamtbetrachtung, die den ganzen Abfall, die unüberschaubare Lawine der ausrangierten, aber funktionstüchtigen Gegenstände mit einbezieht, die also auch die stummen Agenten wie die Rohstoffe und Müllhalden berücksichtigt, wird vermutlich wiederum das Konzept der möglichst ausgedehnten Langlebigkeit mittel- wie kurzfristig die zu wählende Option ausmachen. Dabei ist die neuerliche Forderung nach langlebigen Dingen vielleicht sogar abermals aus dem Mangel heraus geboren: dem Mangel an Entsorgungsstätten für all die kurzlebigen Dinge. ${ }^{47}$

44 Vgl. den Beitrag von Möller in diesem Sonderheft. Zu den sozialistischen Wirtschaftsprinzipien und ihrem Fokus auf Recycling-Möglichkeiten vgl. außerdem Zsuzsa Gille, From the Cult of Waste to the Trash Heap of History. The Politics of Waste in Socialist and Postsocialist Hungary, Bloomington 2000.

45 Vgl. zu den Reparaturbetrieben von gebrauchten Glühlampen Luxbacher (wie Anm. 4), S. $106 \mathrm{ff}$.

46 Vgl. als frühen Vertreter eines derartigen Produktionsregimes auch Stuart Chase, Tragödie der Verschwendung. Gemeinwirtschaftliche Gedanken in Amerika, Oldenbourg u.a.O. 1927.

47 Vgl. hierzu die Einleitung zu dem Sonderheft. 
Die technische Optimierung industrieller Fertigungsprozesse hebt Produkte im frühen 20. Jahrhundert auf ein Qualitätsniveau, das - theoretisch wie praktisch $-\mathrm{zu}$ einer hohen Belastbarkeit und großer Langlebigkeit der Erzeugnisse führen könnte. Dennoch setzte im Fall der Glühlampe ein Forschungsprozess ein, der die Produkte so zu verändern angewiesen war, dass sie nicht mehr das Optimum aus dem Material, sondern aus dem Markt herausholten. Die technische Optimierung hörte also nicht auf, sondern polte nur ihr Ziel um: Die einmal erreichte Langlebigkeit galt es statt zu steigern neu zu kalibrieren auf eine möglichst genau gesteuerte Mittelfristigkeit. Die ungewöhnliche Aufgabe, vor der sich das Phoebus-Kartell und vergleichbare marktbeherrschende Akteure wiederfinden, besteht darin, dass technisch ausgereifte Objekte wie die Glühlampe wieder zu epistemischen Dingen, das heißt zu technisch ungesicherten, instabilen Dingen werden, die sodann darauf zielen, sich in ein ökonomisch kontrolliertes, auto-entropisches Objekt zu verwandeln, das sein Ziel mit dem exakt kalkulierten Kaputtgehen erreicht.

Das „Kaputtgehen“ von Technik, so hat kürzlich Möser formuliert, ist eine ,extreme Form der Nichteinhaltung von Funktionsversprechen“ “. ${ }^{48}$ Auch im Zeitalter der Wegwerfkultur erwarten Nutzer, dass technische Artefakte möglichst lange ihren Dienst tun, und keinerlei Hinweis seitens der Produzenten hält sie von dieser Annahme ab. Eine vorzeitige Dysfunktionalität weicht von dieser Erwartung ab; es stellt also einen Fehler dar. Wie hängt nun dieser planmäßig implantierte Fehler - die gezielt gebaute und zugleich vor dem Verbraucher verborgene Sollbruchstelle im Produkt - mit den innerhalb der Kulturwissenschaften gängigen Theorien des Fehlers zusammen? Greift hier noch die These, dass dem unvorhergesehenen Ereignis, dem Unfall, eine spezifische Produktivität, ${ }^{49}$ ein epistemischer Mehrwert, oder eine katalytische Funktion innewohnt, ${ }^{50}$ insofern der Unfall oder die Störung als Unterbrechung der Routine Anlass geben, die eingespielten Arbeitsabläufe und Konstruktionsweisen zu überdenken, um den derart entdeckten Fehler auszuräumen? Inwiefern also werden hier mit dem Fehler die produktiven Momente des technischen Fortschritts mobilisiert? Die Suggestivität der Fragestellung deutet es bereits an: Weil die gängigen Fehlertheorien, ${ }^{51}$ die darauf angelegt sind, die Störungen zu registrieren, um sie auszuräumen und dem Fortschritt seinen Weg zu bahnen, ${ }^{52}$ hier nicht greifen, muss man von einer anderen Logik ausgehen, mit der bei der industriellen Obsoleszenz der Fehler operationalisiert wird.

48 Vgl. Kurt Möser, Grauzonen der Technikgeschichte, Karlsruhe 2011, S. 95-101, hier S. 97.

49 Vgl. Peter Geimer, Was ist kein Bild? Zur Zerstörung der Verweisung, in: Peter Geimer (Hg.), Ordnungen der Sichtbarkeit. Fotografie in Wissenschaft, Kunst und Technologie, Frankfurt a.M. 2002, S. 313-340.

50 Vgl. dazu Douglas Allchin, Epistemology of Error. Unpublished Typoscript, Vancouver 2000

51 Von den Fehlertheorien in der Mathematik (man denke etwa an die Ausgleichsgerade) oder in der Moralphilosophie soll gar nicht die Rede sein.

52 Packard (wie Anm. 13), Allchin (wie Anm. 50). 
Es findet eine Umkehrung der Entwicklungsrichtung statt: Der Fehler dient nicht als vorausgegangenes, zu analysierendes Element innerhalb eines Ursache-Wirkungs-Zusammenhangs. Vielmehr betreibt die geplante Obsoleszenz eine dezidierte Wirkungs-Ursache-Forschung. Schließlich lautet die Forschungsfrage nicht, wie man durch die Analyse von Wirkungen eine Ursache verhindern kann. Man denke etwa an die Untersuchung von Rissen in den Kacheln des Hitzeschutzschilds des Columbia-Spaceshuttles vor beinahe zehn Jahren, deren Analyse eine Kausalkette von beim Start abgetrennten Schaumstoffteilchen hin zu den Löchern in den Kacheln aufwies. Die Forschungsfrage der geplanten Obsoleszenz lautet stattdessen, wie man gezielt einen Fehler verursachen kann, also, um im Beispiel zu bleiben, wie der Schaumstoff am besten befestigt wird, damit er beim Start so abfällt, dass er die Kacheln beschädigt. Oder anders, wie kann man ein Ereignis so programmieren oder einstellen, dass es wie ein - allerdings Nutzer und Umwelt tunlichst unbeschädigt lassender - technischer Unfall aussieht, und zugleich dazu führt, einen Gegenstand nicht nur - wie Bernard London es forderte - , legally dead", sondern nach ziemlich genau 1.000 Stunden Brenndauer einer Glühlampe, z.B. den Leuchtfaden technisch ,tot ${ }^{6}$ zu machen, während es für den Verbraucher zugleich wie ein Zufall erscheint? Spätestens an diesem Punkt zeigt sich, dass die Technologien des 20. Jahrhunderts neben ihrer vermeintlichen Zentralperspektive einer teleologischen Fortschrittsidee ebenso ihre bisher noch kaum erforschten Gegenprogramme besitzen. Der glückliche Archivfund der aus den 1930er Jahren überlieferten Osram-Akten mit ihrer Geschäftskorrespondenz macht unwiderlegbar klar, wie intensiv die Forschungs- und Entwicklungsanstrengungen im Rahmen der geplanten Obsoleszenz erfolgt sind. Ob und in welcher Form genau sich das damit eingespielte System auch auf andere Branchen übertragen und dort ebenso nachweisen lässt, wird die Technikgeschichtsforschung allerdings anhand weiterer Firmenarchive prüfen müssen.

Ein wesentlicher Grund, warum sich zu Beginn des 20. Jahrhunderts die geplante Obsoleszenz - neben der zeitgleichen Entwicklung der Planwirtschaft - als ein neues Paradigma der technischen Optimierung etablieren kann, in der nun das technisch-wissenschaftlich Erreichbare radikal den ökonomischen Prämissen untergeordnet wird, liegt aber gerade darin, dass die Obsoleszenz im Opaken arbeitet. Anders als die gut sichtbare Tablettenspaltzone wird sie den Blicken des Verbrauchers systematisch entzogen und als „Fehler“ konstruiert, um unter diesem Deckmantel im Verborgenen gegen das Wohl des Konsumenten zu wirken.

Anschrift des Verfassers: Prof. Dr. Markus Krajewski, Universität Basel, s/f/m seminar für medienwissenschaft, Holbeinstrasse 12, CH-4051 Basel, Schweiz 